\title{
EDITORIAL
}

\section{Rhinovirus infections in infants: is respiratory syncytial virus ready for the challenge?}

\author{
N. Regamey* and L. Kaiser\#
}

H uman rhinoviruses are the leading cause of upper respiratory tract infections (i.e. the common cold) in both adults and children [1]. On average, adults experience one rhinovirus infection each year, whereas, in young children, it is a more frequent occurrence. Although rhinovirus infections are limited to the common cold in most cases, they are also a frequent cause of acute sinusitis and otitis media (subsequently leading to antibiotic overuse). Rhinovirus tropism and replication, however, is not limited to the upper respiratory tract. It has been recognised that rhinoviruses can replicate in the lower respiratory tract and lead to lower respiratory tract illnesses, particularly in the elderly, in immunocompromised patients and in children. It is has also been established that rhinoviruses contribute to the majority of asthma exacerbations in both adults and children, and to nearly half of all chronic obstructive pulmonary disease (COPD) exacerbations. Given the technical difficulties involved in isolating rhinovirus in cell cultures, it has only been in recent years, with the advent of viral genome detection via RTPCR, that a clearer understanding of the impact of these infections has emerged [2].

In infants, acute viral respiratory illnesses are the major cause of morbidity and mortality. Several recent community-based studies of infants at high risk of atopy or those attending day care have consistently found rhinoviruses to be the most frequent viruses associated with acute respiratory infections or wheezing illnesses [3-5]. The leading role of rhinoviruses in infancy was recently corroborated in a birth cohort study of otherwise healthy infants, who were studied at the first acute respiratory infection with cough or wheeze [6]. In addition, the potential severity of rhinovirus-associated diseases has been demonstrated by case series describing bronchiolitis, pneumonia and wheezing illnesses in hospitalised infants with rhinovirus infection [7-10].

In this issue of the European Respiratory Journal (ERJ), JARTTI et al. [11] present a study of the viral aetiology of respiratory illnesses in a subgroup of infants of the Childhood Origins of

\footnotetext{
*Dept of Paediatric Respiratory Medicine, University Children's Hospital of Berne, Berne, and ${ }^{\#}$ Central Laboratory of Virology, Division of Infectious Diseases and Division of Laboratory Medicine University Hospital of Geneva, Geneva, Switzerland.

STATEMENT OF INTEREST: None declared.

CORRESPONDENCE: N. Regamey, Dept of Paediatric Respiratory Medicine, University Children's Hospital, Inselspital, 3010 Berne, Switzerland. Fax: 41 316329431. E-mail: Nicolas.Regamey@ insel.ch
}

ASThma (COAST) study, a prospective birth cohort study that enrolled $\sim 300$ newborns from Wisconsin (USA) who were at increased risk of developing asthma (i.e. at least one parent with allergies and/or asthma). The authors focused on infants who were frequently ill (27 infants out of 285), i.e. those who had five or more moderate-to-severe respiratory illnesses during their first year of life. JARTTI et al. [11] found that in this group of patients, rhinoviruses were the most commonly detected viruses in nasal washes (detected in $61 \%$ of cases of moderate-to-severe respiratory illnesses and in $43 \%$ of mild illnesses). Rhinovirus infections occurred early in life, with the mean age at first rhinovirus infection being 4 months. They were usually associated with wheezing, had severity similar to other infections, such as respiratory syncytial virus (RSV), and, interestingly, were the result of reinfections, i.e. two consecutive rhinovirus infections in an infant were usually caused by two different strains and not by a protracted infection with one virus strain.

What are the important messages to take from this study? The study of JARTTI et al. [11] adds to the increasing evidence that rhinoviruses play a so far underestimated role in respiratory morbidity in infancy. Secondly, it demonstrates that at least in the population studied, recurrent rhinovirus infections are not the result of chronic, persistent infections, but are the result of reinfections with different serotypes. Since $>100$ serotypes have been characterised and as most of them circulate in the community, reinfection is expected to occur throughout life. Certainly, some cross-protection does exist between close serotypes but this has an overall limited effect given the large variability of viral capsid protein 1 and other surface viral glycoproteins targeted by the immune response. In addition, new rhinovirus subtypes have been recently identified [12-14], highlighting the potential ability of these viruses to diversify and to escape pre-existing immune responses. Thus, the diversity of circulating strains and the limited ability of previous immunity to protect against reinfection are key determinants to consider for any strategy aimed at preventing or treating rhinovirus infections in infants.

Despite these observations, several questions regarding rhinovirus infections in infancy remain unanswered. Most importantly, the clinical significance of the detection of rhinoviruses in respiratory samples from infants has not yet been clearly established. In the study of JARTTI et al. [11], rhinoviruses were the most common viruses detected not only during periods of illness but also during asymptomatic visits (where they were detected in $35 \%$ of cases). These figures are in agreement with 
other studies, which have found that respiratory specimens from $11-20 \%$ of infants asymptomatic at the time of sampling were positive for rhinovirus [4, 15-17]. These asymptomatic infections may represent remnant RNA from a previous symptomatic infection (although the findings of JARTTI et al. [11] argue against this hypothesis), the beginning of a developing symptomatic infection or they may simply reflect the fact that the spectrum of rhinovirus infections includes episodes with minimal clinical disease. A recent prospective community-based birth cohort study of infants at high risk of atopy provided important information regarding this issue; KUSEL et al. [4] calculated attributable risks for each virus detected, e.g. the proportion of infection attributable to a particular virus. It was found that $47 \%$ of upper respiratory infections and $32 \%$ of lower respiratory infections in infants were attributable to rhinoviruses. In comparison, only 3\% of upper respiratory infections and $10 \%$ of lower respiratory infections were attributable to RSV. Unfortunately, such information is missing from the paper of JARTTI et al. [11], but it could be estimated that roughly a quarter of the moderate-tosevere respiratory illnesses (rhinovirus detection rate in moderate-to-severe respiratory illnesses minus rhinovirus detection rate during asymptomatic visits) were attributable to rhinoviruses.

Current evidence leaves no doubt that rhinoviruses are associated with significant respiratory morbidity in infants. It now remains to be investigated what determines the disease severity in a particular infant. Differences in the virus or the susceptibilities of the host (e.g. pre-existing immunity) may play a role. Recent work has suggested that new rhinovirus subgroups may be associated with specific clinical manifestations [12-14]. However, definitive proof of this concept is lacking, although it is acknowledged that enteroviruses, which share similar genomic organisation, include variants with different tropism and clinical phenotype. In contrast, there is clear evidence that there are genetic, environmental and immunological factors that affect the clinical manifestation of any viral infection. Asthmatic patients, for instance, show an increased susceptibility to viral infections, in particular to rhinoviruses [18, 19]. Whether an increased susceptibility toward rhinovirus infections could play a role in the at-risk population studied by JARTTI et al. [11] has to be considered. Further research should address the relative contribution of viral and host factors and allow a better understanding of the pathophysiological role of rhinovirus infections in infancy.

So what do these findings mean for the paediatrician in clinical practice? Will the detection of rhinoviruses change the management of children with respiratory infections? Although implementation of rapid picornavirus detection assays has been proposed for the clinical diagnosis of respiratory infections in paediatric patients with bronchiolitis [8], the evidence that such a practice will affect clinical management is lacking. Conversely, it is well established that the rapid diagnosis of influenza or RSV infection in children has a significant impact on physician decision-making, and these tests are routinely used [20].

Will "wheeze with rhinovirus" be implemented in algorithms predicting the development of asthma? LEMANSKE et al. [3] have already provided important prognostic information for the practicing physician with regard to this issue. They have shown that in infants at increased risk of developing asthma, the most significant risk factor for the development of preschool wheezing is the occurrence of symptomatic rhinovirus illnesses during infancy; $63 \%$ of infants who wheezed during the rhinovirus season continued to wheeze in the third year of life, compared with $20 \%$ who wheezed during the RSV season. However, these findings and their potential impact on early childhood asthma need to be validated in unselected infant populations. At this stage, it may therefore be too early to draw firm conclusions about the role of rhinovirus diagnosis in daily clinical practice.

In summary, the paper by JARTTI et al. [11] underscores the major role of rhinovirus infections in early life. In the mind of most paediatricians, rhinovirus is not ready to compete with respiratory syncytial virus, the so far unchallenged leader of viral infections in infancy. However, systematic screening for rhinovirus could change perceptions and lead us to revisit this dogma. The underdog is challenging the leader. Paediatricians: be aware and watch out for rhinoviruses!

\section{REFERENCES}

1 Brownlee JW, Turner RB. New developments in the epidemiology and clinical spectrum of rhinovirus infections. Curr Opin Pediatr 2008; 20: 67-71.

2 Garbino J, Gerbase MW, Wunderli W, et al. Lower respiratory viral illnesses: improved diagnosis by molecular methods and clinical impact. Am J Respir Crit Care Med 2004; 170: 1197-1203.

3 Lemanske RF Jr, Jackson DJ, Gangnon RE, et al. Rhinovirus illnesses during infancy predict subsequent childhood wheezing. J Allergy Clin Immunol 2005; 116: 571-577.

4 Kusel MM, de Klerk NH, Holt PG, Kebadze T, Johnston SL, Sly PD. Role of respiratory viruses in acute upper and lower respiratory tract illness in the first year of life: a birth cohort study. Pediatr Infect Dis J 2006; 25: 680-686.

5 Souza LS, Ramos EA, Carvalho FM, et al. Viral respiratory infections in young children attending day care in urban Northeast Brazil. Pediatr Pulmonol 2003; 35: 184-191.

6 Regamey N, Kaiser L, Roiha HL, et al. Viral etiology of acute respiratory infections with cough in infancy: a community-based birth cohort study. Pediatr Infect Dis J 2008; 27: 100-105.

7 Papadopoulos NG, Moustaki M, Tsolia M, et al. Association of rhinovirus infection with increased disease severity in acute bronchiolitis. Am J Respir Crit Care Med 2002; 165: 1285-1289.

8 Jacques J, Bouscambert-Duchamp M, Moret $\mathrm{H}$, et al. Association of respiratory picornaviruses with acute bronchiolitis in French infants. J Clin Virol 2006; 35: 463-466.

9 Miller EK, Lu X, Erdman DD, et al. Rhinovirus-associated hospitalizations in young children. J Infect Dis 2007; 195: 773-781.

10 Calvo C, Garcia-Garcia ML, Blanco C, Pozo F, Flecha IC, Perez-Brena P. Role of rhinovirus in hospitalized infants with respiratory tract infections in Spain. Pediatr Infect Dis J 2007; 26: 904-908.

11 Jartti T, Lee W-M, Pappas T, Evans M, Lemanske RF Jr, Gern JE. Serial viral infections in infants with recurrent respiratory illnesses. Eur Respir J 2008; 32: 314-320. 
12 Lamson D, Renwick N, Kapoor V, et al. MassTag polymerase-chain-reaction detection of respiratory pathogens, including a new rhinovirus genotype, that caused influenza-like illness in New York State during 2004-2005. J Infect Dis 2006; 194: 1398-1402.

13 Renwick N, Schweiger B, Kapoor V, et al. A recently identified rhinovirus genotype is associated with severe respiratory-tract infection in children in Germany. J Infect Dis 2007; 196: 1754-1760.

14 McErlean P, Shackelton LA, Andrews E, et al. Distinguishing molecular features and clinical characteristics of a putative new rhinovirus species, human rhinovirus C (HRV C). PLoS ONE 2008; 3: e1847.

15 van Benten I, Koopman L, Niesters B, et al. Predominance of rhinovirus in the nose of symptomatic and asymptomatic infants. Pediatr Allergy Immunol 2003; 14: 363-370.
16 Nokso-Koivisto J, Kinnari TJ, Lindahl P, Hovi T, Pitkaranta A. Human picornavirus and coronavirus RNA in nasopharynx of children without concurrent respiratory symptoms. J Med Virol 2002; 66: 417-420.

17 Jartti T, Lehtinen P, Vuorinen T, Koskenvuo M, Ruuskanen O. Persistence of rhinovirus and enterovirus RNA after acute respiratory illness in children. J Med Virol 2004; 72: 695-699.

18 Corne JM, Marshall C, Smith S, et al. Frequency, severity, and duration of rhinovirus infections in asthmatic and non-asthmatic individuals: a longitudinal cohort study. Lancet 2002; 359: 831-834.

19 Contoli M, Message SD, Laza-Stanca V, et al. Role of deficient type III interferon- $\lambda$ production in asthma exacerbations. Nat Med 2006; 12: 1023-1026.

20 Charles PG. Early diagnosis of lower respiratory tract infections (point-of-care tests). Curr Opin Pulm Med 2008; 14: 176-182. 